\title{
Two Problems on Coinvariant Subspaces of the Shift Operator
}

\author{
Konstantin M. Dyakonov

\begin{abstract}
Two problems are posed that involve the star-invariant subspace $K_{\theta}^{p}$ (in the Hardy space $H^{p}$ ) associated with an inner function $\theta$. One of these asks for a characterization of the extreme points of the unit ball in $K_{\theta}^{\infty}$, while the other concerns the Fermat equation $f^{n}+g^{n}=h^{n}$ in $K_{\theta}^{p}$.
\end{abstract}

Mathematics Subject Classification (2010). 30H05, 30H10, 30J05, 47 B35.

Keywords. Hardy spaces, star-invariant subspaces, extreme points, Fermat's equation.

Let $H^{p}$ stand for the classical Hardy space on the disk $\mathbb{D}:=\{z \in \mathbb{C}$ : $|z|<1\}$. As usual, we identify $H^{p}$-functions with their boundary values and treat $H^{p}$ as a subspace of $L^{p}(\mathbb{T}, m)$; here $\mathbb{T}:=\partial \mathbb{D}$ is the unit circle and $m$ is the normalized arclength measure on $\mathbb{T}$. The shift operator $S: H^{p} \rightarrow H^{p}$ acts by the rule $(S f)(z)=z f(z)$, and we know from Beurling's theorem that the nontrivial $S$-invariant subspaces in $H^{p}$, with $p \in(0, \infty)$, are precisely those of the form $\theta H^{p}$, where $\theta$ is an inner function. (By definition, an inner function is an $H^{\infty}$-function whose modulus equals 1 a.e. on $\mathbb{T}$.) For Beurling's theorem and other basic facts about $H^{p}$ spaces, see [5, Chap. II].

Now, the $S$-coinvariant (or star-invariant, or model) subspace generated by an inner function $\theta$ is

$$
K_{\theta}^{p}:=H^{p} \cap \theta \overline{H_{0}^{p}},
$$

where $H_{0}^{p}=z H^{p}$. Here and below, we restrict ourselves to the range $p \in$ $[1, \infty]$, and we endow $K_{\theta}^{p}$ with the usual $H^{p}$-norm $\|\cdot\|_{p}$. Such a subspace is invariant under the backward shift operator $S^{*}: f \mapsto(f-f(0)) / z$ on $H^{p}$ and, for $p$ finite, it provides the general form of an $S^{*}$-invariant subspace. This last statement is easy to deduce, at least for $p \in(1, \infty)$, from Beurling's theorem by duality, since $K_{\theta}^{p}$ is the annihilator (in $H^{p}$ ) of the $S$-invariant subspace $\theta H^{q}$ (in $H^{q}$, with $p^{-1}+q^{-1}=1$ ) with respect to the standard

Supported in part by grant MTM2011-27932-C02-01 from El Ministerio de Ciencia e Innovación (Spain) and grant 2009-SGR-1303 from AGAUR (Generalitat de Catalunya). 
pairing $\langle f, g\rangle=\int_{\mathbb{T}} f \bar{g} d m$. In particular, we have $K_{\theta}^{2}=H^{2} \ominus \theta H^{2}$. We finally observe that, for $p \in[1, \infty]$ and $\theta$ inner, the subspace $K_{\theta}^{p}$ coincides with the kernel of $T_{\bar{\theta}}$, the Toeplitz operator with symbol $\bar{\theta}$, in $H^{p}$.

This note offers two open problems on $K_{\theta}^{p}$ spaces that were previously posed by the author at the workshop "Operator Theory and Harmonic Analysis" in Oberwolfach, Germany (November 2010) and at the CRM conference "Invariant Subspaces of the Shift Operator" in Montreal, Canada (August 2013).

When stating the first of these, and later on, we write ball $(X)$ to denote the closed unit ball of a Banach space $X$.

Problem 1. Given an inner function $\theta$, characterize the extreme points of $\operatorname{ball}\left(K_{\theta}^{\infty}\right)$.

One may begin by recalling that the extreme points of ball $\left(H^{\infty}\right)$ are precisely the unit-norm functions $f \in H^{\infty}$ with

$$
\int_{\mathbb{T}} \log (1-|f|) d m=-\infty ;
$$

see [1] or [5, Chap. IV]. It follows that every non-inner function in ball $\left(K_{\theta}^{\infty}\right)$ will be non-extreme for ball $\left(H^{\infty}\right)$. Indeed, for $f \in K_{\theta}^{\infty}$, we have

$$
\left(1-|f|^{2}\right) \theta=\theta-f \cdot \bar{f} \theta \in H^{\infty},
$$

because $\bar{f} \theta \in H^{\infty}$. Assuming that $|f| \leq 1$ and $|f| \not \equiv 1$ on $\mathbb{T}$, we see from (2) that $1-|f|^{2}$ agrees with the modulus of a non-null $H^{\infty}$-function. Therefore, $\log \left(1-|f|^{2}\right) \in L^{1}(\mathbb{T})$ and the integral in (11) is convergent.

On the other hand, the space $K_{\theta}^{\infty}$ (and its unit ball) will contain inner functions if and only if $\theta(0)=0$. Those inner functions are then precisely the divisors of $\theta / z$, and they are sure to be extreme points for ball $\left(K_{\theta}^{\infty}\right)$. It is the non-inner extreme points of this ball that we are concerned with.

In [3, Problem 1 was solved in the simplest case where $\theta(z)=z^{N+1}$, with $N \in \mathbb{N}$. In this case, $K_{\theta}^{\infty}$ becomes the space of polynomials of degree at most $N$, endowed with the sup-norm; we shall denote the latter space by $\mathcal{P}_{N}$. The solution given in $[3$ is, however, less elementary than one might at first expect. Namely, a non-inner (or equivalently, non-monomial) unitnorm polynomial $P \in \mathcal{P}_{N}$ is shown to be extreme for ball $\left(\mathcal{P}_{N}\right)$ if and only if a certain Wronski-type matrix built from $P$ has maximal rank. It turns out that this rank condition cannot be rephrased, in any reasonably explicit way, in terms of the "smallness" or the zeros of $1-|P|^{2}$. In fact, a simple construction from [3] produces two unit-norm polynomials, $P$ and $Q$, in $\mathcal{P}_{N}$ satisfying $1-|P|^{2}=2\left(1-|Q|^{2}\right)$ and such that $P$ is a non-extreme point of $\operatorname{ball}\left(\mathcal{P}_{N}\right)$, while $Q$ is extreme.

The case of $\mathcal{P}_{N}$, let alone $K_{\theta}^{\infty}$ with a general $\theta$, therefore exhibits a higher level of complexity than $H^{\infty}$ (where the extreme points are described by (11) or the space of real-valued trigonometric polynomials $P$ of degree $\leq N$ (where the description involves only the number of zeros of $1-|P|^{2}$ on $\mathbb{T}$, see $[3,47,8])$. When $\theta$ is a finite Blaschke product, the extreme points of 
ball $\left(K_{\theta}^{\infty}\right)$ are probably describable in the spirit of [3], but the general case seems to call for a new technique.

Before moving on to the next problem, we mention that the extreme points of ball $\left(K_{\theta}^{1}\right)$ were characterized in 2. A more general result was actually proved there, the space in question being the kernel (in $H^{1}$ ) of an arbitrary Toeplitz operator $T_{\varphi}$ with $\varphi \in L^{\infty}$. When specialized to the case $\varphi=\bar{\theta}$, with $\theta$ inner, the result tells us that a unit-norm function $f \in K_{\theta}^{1}$ is an extreme point of ball $\left(K_{\theta}^{1}\right)$ if and only if the inner factors of $f$ and $\bar{z} \bar{f} \theta$ are relatively prime. This can be viewed as an extension of de Leeuw and Rudin's theorem that identifies the extreme points of ball $\left(H^{1}\right)$ as outer functions; see [1] or [5, Chap. IV].

Problem 2. Prove or disprove "Fermat's last theorem" for $K_{\theta}^{p}$ : whenever $\theta$ is inner, $p \in[1, \infty]$ and $n \in\{3,4,5, \ldots\}$, no nontrivial triple of functions $f, g, h \in K_{\theta}^{p}$ satisfies $f^{n}+g^{n}=h^{n}$.

Here, by calling a triple $(f, g, h)$ nontrivial we mean that the three functions do not lie in a single one-dimensional subspace of $K_{\theta}^{p}$.

In the polynomial case (i.e., for $\theta(z)=z^{N+1}$ ), Fermat's last theorem is known to be true: the equation $P^{n}+Q^{n}=R^{n}$ has no nontrivial polynomial solutions when $n \geq 3$. (Such solutions do exist for $n=2$, as simple examples show.) It follows, then, that a similar result holds with rational functions in place of polynomials, and this settles Problem 2 in the case where $\theta$ is a finite Blaschke product. The polynomial Fermat theorem, as stated above, is best proved as a consequence of the Mason-Stothers "abc theorem" (the prototype of the famous "abc conjecture" in number theory); the deduction consists in a three-line argument that can be found in [4, 6, 9]. Now, the Mason-Stothers theorem provides a lower bound on the number of distinct zeros of the polynomial $P Q R$ in terms of the degrees of $P, Q$ and $R$. In 4, we obtained some estimates of a similar nature for general holomorphic functions on the disk, while the case of entire functions was treated earlier in [6]. It seems that a suitable $K_{\theta}^{p}$ version of the Mason-Stothers theorem, if available, might give us a clue to Problem 2.

\section{References}

[1] K. de Leeuw and W. Rudin, Extreme points and extremum problems in $H_{1}$. Pacific J. Math. 8 (1958), 467-485.

[2] K. M. Dyakonov, Interpolating functions of minimal norm, star-invariant subspaces, and kernels of Toeplitz operators. Proc. Amer. Math. Soc. 116 (1992), $1007-1013$.

[3] K. M. Dyakonov, Extreme points in spaces of polynomials. Math. Res. Lett. 10 (2003), 717-728.

[4] K. M. Dyakonov, Zeros of analytic functions, with or without multiplicities. Math. Ann. 352 (2012), 625-641.

[5] J. B. Garnett, Bounded analytic functions, Revised first edition. Springer, New York, 2007. 
[6] G. G. Gundersen and W. K. Hayman, The strength of Cartan's version of Nevanlinna theory. Bull. London Math. Soc. 36 (2004), 433-454.

[7] A. G. Konheim and T. J. Rivlin, Extreme points of the unit ball in a space of real polynomials. Amer. Math. Monthly 73 (1966), 505-507.

[8] H.-J. Rack, Extreme Punkte in der Einheitskugel des Vektorraumes der trigonometrischen Polynome. Elem. Math. 37 (1982), 164-165.

[9] T. Sheil-Small, Complex polynomials. Cambridge Studies in Advanced Mathematics, 75, Cambridge University Press, Cambridge, 2002.

Konstantin M. Dyakonov

ICREA and Universitat de Barcelona

Departament de Matemàtica Aplicada i Anàlisi

Gran Via, 585

E-08007 Barcelona

Spain

e-mail: konstantin.dyakonov@icrea.cat 